Caries Res 1996:30:473-474

\title{
Subject Index Vol. 30, 1996
}

\section{Acid etch 83}

Acidogenic potential index 342

Acidogenicity 434

Actinomyces naeslundii 40

Adolescence 29, 34

Adolescents 143

Adsorption 119

Adults 101,342

Aged 169

AgNOR 225

Ammonium hexafluorosilicate 465

Amylase 65

Animal model, rat 163

Anticariogenic agent 119

Artificial caries lesions 400

Atraumatic restorative treatment 428

Bicarbonate 361 Bitewing image 143

- radiography 156

Blackcurrant drink 342

Blacks 169

Bulimia nervosa 361

Calcium 71,458

phosphates 213 Caries 8, 101,231,334

activity 143

experience 29

experimental 367

incidence 256

increments 408

prediction 256

- factors 418

prevalence 237

prevention 418

trend 423 Cariogenicity 126 Cavitation 143,313 Cereals 132 Chewing gum 408 Children 8, 16, 342 Chlorhexidine 40, 45, 445 Citric acid 213

Class II amalgam restoration 189 Clinical examination 1

Coffee 138 trial 418,445

Colonization 119

Compliance 445 
Confocal microscopy 317

Consumption, sweets 29

Copolymer devices 200

Copper 367 Coronal caries 1

Demineralisation 45, 93, 126, 326, 373,

394 Dental attrition 163

care 101

caries 16,22,156,225,347,381,428

enamel 373

erosion 163,373

fractures 101

plaque 65, 180, 194

pH 439

radiography 156,389 Dentine 52,76,204,218,231

fluorescence 93 Diagnosis 148,389 Diagnostic methods 381 Diagnostics 93

DMF teeth 22 DMFT 237 dmft 237

Electrical resistance 148 Enamel 76,204

caries 143,317

erosion 213

human 83

hypoplasia 8 Epidemiology 101, 334, 423 Erosion 361

Ethnicity 334

Fissures 231

Fluoridation 76, 347, 465

Fluoride 45, 76, 83, 88, 163, 204, 218, 458

controlled release 200

dentifrice 194

mouth rinse 194

prophylaxis 29

release 454

uptake 454

varnish 347 Fluoroapatite 465 Follow-up study 169 Fructanase 65 Fruits 132 Funoran 119

Gas chromatography 454 Gingival recession 169 Glass ionomer(s) 428

- $\quad$ cement 454

Glucan from sucrose 65 Glucanase 65 Glucosyltransferase 354 Grey discolouration 189

Health behavior 34

- $\quad$ habits 34

Human dental pulp 225

Hydroxyapatite 354, 465

Immigrant 256 Infrared 394 Interdental sites 194 Intra-oral cariogenicity 434

- $\quad$ devices 200

In vitro caries monitoring 394

- $\quad$ microbial model 112

Ionized calcium 213

Lactobacilli 22 Lactobacillus caseí 112,180 Liquid oral medicines 16 Low birth weight 8 
Magnesium 71,458 Maltodextrins 439 Microbiology 52 Microfocal radiography 148 Microprobe analysis 204 Microradiography 317,400 Microsampling 76 Milk, bovine 434

- $\quad$ human 434

Minimal intervention 428

Mutans streptococci 22, 40, 119, 256, 347, 445

Nutritional deficiency 8

Occlusal caries 148,389 Oral hygiene 29

- $\quad$ streptococci 40

Organic materials 218

Pellicle 354 pH 361 Plaque 342

ecology 65

pH 126,132,138,342 Polymers from glucose 65 Posterior teeth 313 Post-fluoridation 204 Prefluoridation 204

473

Preschool children 256,347 Primary teeth 408 Progression 326,381 Proliferating cell nuclear antigen 225

Radiography 326 Radiolucency 313 Rat caries 367 Regression 381 Remineralisation 218,394 Reproducibility 1,326 Restoration(s) 231

- $\quad$ fractures 101

Risk of developing caries 22 Root caries 1, 52, 218

- $\quad$ epidemiology 169

Saliva 180,354

Salivary carbohydrate 132

flow 361

rate 22

pellicle 454

tests 22

Scanning electron microscopy 163 Sealants 428 Secondary caries 112

diagnosis 189 Sialochemistry 180 Side effects 445 Sodium hypochlorite 218

trimetaphosphate 418 Soft drinks 373 Sorbitol 180,408 Specular reflection 394 Streptococcus mutans 112, 180, 458

sanguis 71 Sucralose 138,439 Sucrose 180,367

intake 65

Sulfated polysaccharide 119 Surfaces 354 Sustained release 445 Sweeteners 439

Tea 439

- $\quad$ caffeinated, decaffeinated, herbal

Teeth 204 Tooth staining 445 Toothbrushing 194 Toothpaste 418

Unerupted teeth 83

Validity 326 Varnish(es) 40, 45 Viable counts 65 Virulence factors 65 Viscosity 361 Vomiting 361

Water rinsing 194 - supply 83 Whites 169

Xylitol 34, 180,408

Zinc 71,458

ORCA Announcement

ORCA Rolex Prize 1996 
The 1996 ORCA Rolex Prize has been awarded to Professor Andrew Rugg-Gunn from the University of Newcastle-upon-Tyne, UK

Professor Rugg-Gunn has been and remains an active investigator in the areas of dental caries and dental fluorosis. His scholarly contributions to these subjects are widely recognised and highly respected. He is a tireless contributor to his profession and the public, having served on the editorial boards of Community Dental Health and the British Dental Journal and as a consultant to or member of several government committees concerned with human health. Professor Rugg-Gunn has also been a consistent advocate for ORCA, and ORCA is particularly grateful for his work in relation to the organisation of ORCA Summer Schools. 474

Subject Index Vol. 30, 1996 